\title{
Commentary: Tick, tock ... Time windows for intervention for stroke after cardiac surgery
}

\author{
Juan Marcano, MD, and Ravi K. Ghanta, MD
}

\footnotetext{
From the Division of Cardiothoracic Surgery, Michael E. DeBakey Department of Surgery, Baylor College of Medicine, Houston, Tex.

Disclosures: Authors have nothing to disclose with regard to commercial support.

Received for publication Dec 16, 2018; accepted for publication Dec 17, 2018; available ahead of print Jan 21 2019.

Address for reprints: Ravi K. Ghanta, MD, Michael E. DeBakey Department of Surgery, One Baylor Plaza, MS 390, Houston, TX 77030-3411 (E-mail: Ravi.ghanta@bcm.edu).

J Thorac Cardiovasc Surg 2019;158:199

$0022-5223 / \$ 36.00$

Copyright (c) 2018 by The American Association for Thoracic Surgery

https://doi.org/10.1016/j.jtcvs.2018.12.053
}

Stroke after cardiac surgery is a devastating complication and a frequently cited disadvantage of cardiac operations to percutaneous interventions. Because the atherosclerotic process is not limited to the heart and great vessels, a higher prevalence of patients undergoing cardiac surgery have concomitant cerebrovascular disease. In addition, embolic risk is present with all cardiac surgery interventions. Consequently, much has been invested in reducing neurologic complications, with stroke now a rare occurrence $(1.3 \%)$ after coronary artery bypass grafting. ${ }^{1}$ Nonetheless, the ability to rescue patients who experience postoperative stroke remains a key interest.

Contemporary advances in neurointervention have made rescue a real possibility. However, the optimal time for intervention is a last seen well (LSW) time $<6$ hours, which is not practical for patients who undergo lengthy cardiac operations or have a more complex initial postoperative course. In many institutions, these patients are not offered neurointervention. In a new case series, Sheriff and colleagues ${ }^{2}$ challenge the dogma of restricting intervention to early time windows. They present a single-institution experience from the Massachusetts General Hospital of 7 patients who underwent postcardiac surgery neurointervention for stroke from 2013 to 2018. The patients experienced stroke associated with large vessel occlusion, defined as occlusion or either terminal internal carotid or proximal middle cerebral artery or basilar artery found on computed tomography angiography or magnetic resonance angiography. All patients were evaluated for mechanical thrombectomy using an established institutional protocol. The patients were designated into 2 categories: early (LSW $\leq 6$ hours $[\mathrm{n}=5]$ ) and late (LSW $\geq 6$ hours $[\mathrm{n}=2]$ ). Patients in both groups demonstrated improvement in stroke scores and better functional outcome at 3 months postoperatively. They identified no major iatrogenic complication secondary to intervention. Although the Sheriff and colleagues ${ }^{2}$ series represents a small and heterogeneous sample, it does illustrate the potential benefit of intervention for this potentially devastating complication. They also evaluate risk factors

\section{References}

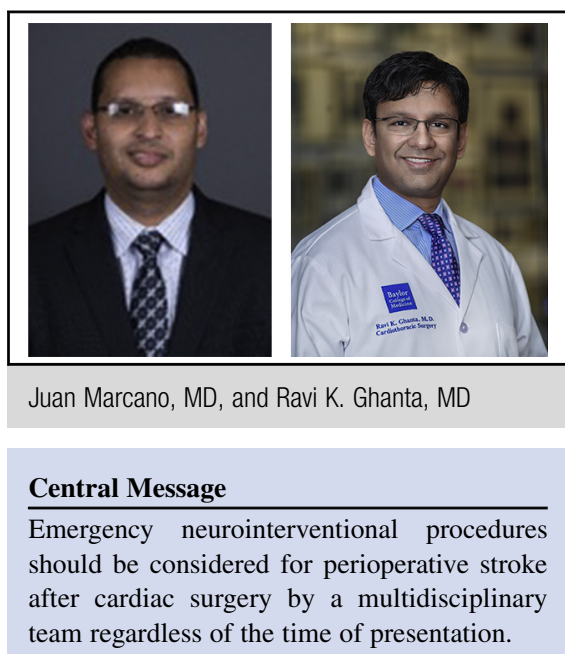

See Article page 186

for stroke. They identified a $2.4 \%$ incidence of ischemic strokes where $10 \%$ was secondary to large vessel occlusion. Univariate analysis showed increased risk with pump times beyond 150 minutes and crossclamp times more than 110 minutes. Vascular risk factors identified included hypertension $(69.9 \%)$, hyperlipidemia $(59.9 \%)$, smoking $(52.1 \%)$, atrial fibrillation $(28.1 \%)$, and prior stroke $(13.7 \%)$, similar to prior studies. ${ }^{3}$

Sheriff and colleagues ${ }^{2}$ are to be congratulated for evaluating their experience of high-risk neurointervention in patients after cardiac surgery. A recent trial also demonstrated the benefit of intervention in patients beyond the 6-hour window. ${ }^{4}$ This study further highlights the importance of multidisciplinary evaluation of patients with neurological deficits after cardiac surgery and the possibility of rescue. The clock is ticking in patients who experience stroke, but the window of time for rescue may be expanding.

1. D'Agostino RS, Jacobs JP, Badhwar V, Fernandez FG, Paone G, Wormuth DW, et al. The Society of Thoracic Surgeons Adult Cardiac Surgery Database: 2019 up date on outcomes and quality. Ann Thorac Surg. 2019;107:24-32.

2. Sheriff F, Hisch JA, Shelton K, D’Allesandro D, Stapleton C, Koch M, et al. Large vessel occlusion stroke after cardiothoracic surgery: expanding time windows offer new salvage opportunities. J Thorac Cardiovasc Surg. 2019;158:186-96.e2.

3. Tarakji KG, Sabik JF III, Bhudia SK, Batizy LH, Blackstone EH. Temporal onset, risk factors, and outcomes associated with stroke after coronary artery bypass grafting. JAMA. 2011;305:381-90.

4. Nogueira RG, Jadhav AP, Haussen DC, Bonafe A, Budzik RF, Bhuva P, et al. Thrombectomy 6 to 24 hours after stroke with a mismatch between deficit and infarct. N Engl J Med. 2018;378:11-21. 